Enferm Bras 2020;19(6):518-24

https://doi.org/10.33233/eb.v19i6.4422

\title{
REVISÃo \\ Medidas não-farmacológicas para alívio da dor de recém-nascidos em unidade de terapia intensiva neonatal
}

Thatiane Monick de Souza Costa*, Jessica Cristhyanne Peixoto Nascimento*, Rodrigo Rhuan Andrade Rocha ${ }^{* *}$, Eloysa dos Santos Oliveira**, Bruna Vilar Soares da Silva ${ }^{* * *}$, Evelin Beatriz Bezerra de Melo***, Rodrigo Assis Neves Dantas, D.Sc. ${ }^{* \star *}$, Daniele Vieira Dantas, D.Sc. ${ }^{* \star *}$

${ }^{*}$ Enfermeira, mestranda pelo Programa de Pós-Graduação em Enfermagem da UFRN, ${ }^{*}$ Curso de Graduação em Enfermagem da UFRN, bolsista de iniciação científica PIBIC/UFRN, ${ }^{* * * G r a d u a n d a}$ do Curso de Enfermagem da UFRN, bolsistas de iniciação científica PIBIC/UFRN, ****Enfermeiros, pós-doutores pelo Programa de Pós-Graduação em Enfermagem da Universidade Federal de Sergipe (UFS) e professores adjuntos da Universidade Federal do Rio Grande do Norte (UFRN)

Recebido em 21 de outubro de 2020; aceito em 4 de novembro de 2020.

Correspondência: Rodrigo Assis Neves Dantas, Departamento de Enfermagem da Universidade Federal do Rio Grande do Norte, Natal RN

Thatiane Monick de Souza Costa: thatianemonick@hotmail.com

Jessica Cristhyanne Peixoto Nascimento: jessicacristhy@gmail.com

Rodrigo Rhuan Andrade Rocha: rodrigo.andrade.rocha@hotmail.com

Eloysa dos Santos Oliveira: eloysasantos18@hotmail.com

Bruna Vilar Soares da Silva: bbrunavilar@hotmail.com

Evelin Beatriz Bezerra de Melo: evelinbtz@gmail.com

Rodrigo Assis Neves Dantas: rodrigoenf@yahoo.com.br

Daniele Vieira Dantas: daniele00@hotmail.com

\section{Resumo}

Introdução: Os recém-nascidos em Unidades de Terapia Intensiva são expostos a diversos procedimentos dolorosos. Diante disso, os profissionais deste setor devem identificar e avaliar a dor neonatal. Objetivo: Analisar o contexto das medidas não-farmacológicas para alívio da dor de recém-nascidos, internados em unidades de terapia intensiva neonatal, baseado no referencial teórico de Hinds, Chaves e Cypress. Métodos: Estudo teórico-reflexivo sobre a análise contextual de um fenômeno de enfermagem, partindo da revisão narrativa da literatura científica. Resultados: Observaram-se quatro níveis contextuais sobre medidas nãofarmacológicas aliviadoras da dor neonatal em unidades de terapia intensiva neonatal: aplicação de medidas não-farmacológicas (imediato); elementos influenciadores da aplicação de medidas não-farmacológicas (específico); repercussões dos pais e neonatos diante de aplicação de medidas não-farmacológicas (geral) e legislação sobre o manejo da dor (metacontexto). Conclusão: Possibilitou-se maior entendimento sobre aspectos contextuais das medidas nãofarmacológicas aliviadoras da dor neonatal e melhor compreensão das perspectivas profissionais sobre dificuldades aplicacionais dessas intervenções, evidenciando-se a necessidade de implementar protocolos para avaliação e tratamento da dor, baseados no cuidado integral.

Palavras-chave: dor, manejo da dor, recém-nascido, unidades de terapia intensiva neonatal.

\section{Abstract \\ Non-pharmacological measures for pain relief of newborns in a neonatal intensive care unit}

Background: Newborns in Intensive Care Units are exposed to several painful procedures. Therefore, professionals of these sectors must identify and assess neonatal pain. Objective: To analyze the context of non-pharmacological measures for pain relief in newborns admitted to neonatal intensive care units based on the theoretical framework of Hinds, Chaves and Cypress. Methods: Theoretical-reflective study on the contextual analysis of a nursing phenomenon, based on a narrative review of the scientific literature. Results: Four contextual levels were observed on non-pharmacological measures to relieve neonatal pain in neonatal intensive care units: 
application of non-pharmacological measures (immediate); elements influencing the application of non-pharmacological measures (specific); effects on parents and newborns when applying non-pharmacological measures (general); legislation on pain management (metacontext). Conclusion: A greater understanding of contextual aspects of non-pharmacological measures relieving neonatal pain was made possible; a better understanding of the professional perspectives on application difficulties of these interventions, surfaced the need to implement protocols for pain assessment and treatment based on comprehensive care.

Keywords: pain, pain management, newborn, neonatal intensive care units.

\section{Resumen \\ Medidas no farmacológicas para el alivio del dolor de los recién nacidos en una unidad de cuidados intensivos neonatales}

Introducción: Los recién nacidos en unidades de cuidados intensivos están expuestos a varios procedimientos dolorosos. Por tanto, los profesionales de este sector deben identificar y evaluar el dolor neonatal. Objetivo: Analizar el contexto de las medidas no farmacológicas para el alivio del dolor de los recién nacidos ingresados en Unidades de Cuidados Intensivos Neonatales a partir del marco teórico de Hinds, Chaves y Cypress. Métodos: Estudio teórico-reflexivo sobre el análisis contextual de un fenómeno de enfermería, basado en la revisión narrativa de la literatura científica. Resultados: Se observaron cuatro niveles contextuales sobre medidas no farmacológicas para aliviar el dolor neonatal en Unidades de Cuidados Intensivos Neonatales: aplicación de medidas no farmacológicas (inmediata); elementos que influyen en la aplicación de medidas no farmacológicas (específicas); repercusiones de padres y recién nacidos al aplicar medidas no farmacológicas (general); legislación sobre el manejo del dolor (metacontexto). Conclusión: Se posibilitó una mayor comprensión de los aspectos contextuales de las medidas no farmacológicas que alivian el dolor neonatal, una mejor comprensión de las perspectivas profesionales sobre las dificultades de aplicación de estas intervenciones, evidenciando la necesidad de implementar protocolos de evaluación y tratamiento del dolor, basados en la atención integral.

Palabras-clave: dolor, el manejo del dolor, recién nacido, unidades de cuidados intensivos neonatales.

Introdução

As Unidades de Terapia Intensiva Neonatal (UTIN) são ambientes de grande desenvolvimento e aparato tecnológico, com sofisticados recursos terapêuticos, proporcionando meios para o prolongamento da vida dos recém-nascidos (RN). Porém, esses indivíduos são expostos a uma gama de procedimentos dolorosos realizados diariamente pelos profissionais da saúde. Estima-se que, ao longo do período de internação na UTIN, os neonatos prematuros são submetidos a uma média de 100 procedimentos estressores [1].

A avaliação e identificação da dor necessita ser o foco prioritário da equipe que trabalha em UTIN, pois além da assistência integral à saúde neonatal, adequada e ética, ser um direito básico, também serve para reduzir a exposição desses $\mathrm{RN}$ ao manuseio excessivo e procedimentos desnecessários que geram consequências danosas no desenvolvimento e afetam seu sistema neurológico. Entretanto, nota-se que medidas para o alívio da dor são atípicas no ambiente hospitalar. Tendo isso em vista, faz-se necessário a implementação de métodos capazes de possibilitar o abrandamento do desconforto causado por essas técnicas dolorosas [1-2].

O tratamento não farmacológico tem por objetivo a redução dos estímulos agressivos do ambiente, a prevenção de alterações fisiológicas e comportamentais, provocando alento e diminuição do estresse gerado no RN. Dentre as vantagens, pode-se destacar: baixo custo, facilidade de administração da terapêutica e efeito analgésico. As principais medidas utilizadas são as substâncias adocicadas, especialmente a glicose e a sacarose [3].

Nessa perspectiva, com o propósito de promover uma imersão sobre a temática das medidas não-farmacológicas para alívio da dor neonatal em UTIN, como também corroborar com uma compreensão dos diversos fatores que influenciam essa prática, elaborou-se o seguinte questionamento: Quais os aspectos contextuais que permeiam a aplicação de medidas nãofarmacológicas para alívio da dor de recém-nascidos em UTIN?

O presente estudo teve como objetivo analisar o contexto das medidas nãofarmacológicas para alívio da dor de recém-nascidos internados em Unidade de Terapia Intensiva Neonatal com base no referencial teórico de Hinds, Chaves e Cypress. 
Trata-se de estudo teórico-reflexivo que utilizou como referencial teórico a Análise de Contexto (AC) de Hinds, Chaves e Cypress [4]. A análise contextual foi desenvolvida a partir de revisão narrativa de literatura científica, com caráter qualitativo, realizada em março de 2020 . Os estudos foram organizados e categorizados conforme semelhança e complementaridade de discursos sobre a temática.

A seleção das publicações atendeu aos seguintes critérios de inclusão: artigos científicos disponíveis online e gratuitamente na íntegra via Comunidade Acadêmica Federada (CAFe), portarias ministeriais, guidelines, publicadas no período de 2009 a 2020 e sem restrição de idioma. Foram arroladas publicações em maioria no modelo de artigos científicos, adicionados de decretos e portarias do Ministério da Saúde que abrangeram a temática abordada nesta pesquisa. Foram excluídos artigos que não respondessem o objetivo do estudo, artigos duplicados, resumos e anais de congressos.

É importante salientar que a CAFe é um recurso disponibilizado pelo Portal de Aperfeiçoamento de Pessoal de Nível Superior (CAPES), para busca e acesso de periódicos por meio institucional, na qual a identificação utilizada foi a Universidade Federal do Rio Grande do Norte (UFRN).

Para busca nas fontes de dados, foram selecionados os descritores controlados dos Descritores em Ciências da Saúde (DeCS) - dor; manejo da dor; recém-nascido; unidades de terapia intensiva neonatal - e do Medical Subject Headings (MeSH), a saber: pain; pain management; infant, newborn; intensive care units, neonatal que foram cruzados por meio do operador booleano AND, que resultaram na seleção de 395 artigos para leitura de títulos. A partir da leitura na íntegra, foram selecionados 11 estudos que tinham relação direta com o tema.

O contexto como fenômeno para análise do conhecimento de uma realidade está dividido em quatro níveis: contexto imediato (define os aspectos relevantes da situação), contexto específico (engloba o passado imediato e aspectos importantes da atual situação), contexto geral (interpretações e significados dos indivíduos obtidos através de interações passadas e atuais) e metacontexto (englobam as três dimensões de contextos e congregam aspectos sociopolíticos e normorreguladores que agem sobre o fenômeno) [4].

No sentido de facilitar o estudo, a perspectiva contextual organizou-se da seguinte forma: Contexto imediato: aplicação de medidas não-farmacológicas para alívio da dor de recémnascidos em unidades de terapia intensiva neonatal; Contexto específico: elementos que influenciam a aplicação de medidas não-farmacológicas para alívio da dor de recém-nascidos em unidades de terapia intensiva neonatal; Contexto geral: repercussões dos pais e neonatos diante da aplicação de medidas não-farmacológicas para alívio da dor; Metacontexto: legislação sobre o manejo da dor neonatal.

\section{Resultados e discussão}

Aplicação de medidas não-farmacológicas para alívio da dor de recém-nascidos em unidades de terapia intensiva neonatal

Medidas não-farmacológicas são consideradas intervenções seguras, de fácil aplicação, facilmente acessíveis e eficientes no tratamento da dor em recém-nascidos. Existem diversos métodos para alívio da dor neonatal, sendo os mais utilizados a sacarose ou glicose oral, aleitamento materno, método canguru (cuidados pele a pele), dobra ou desdobramento facilitado, sucção não nutritiva e posicionamento [5].

O aleitamento materno, um dos métodos mais utilizados, é um fenômeno fisiológico, natural e pode ser utilizado como mecanismo eficaz para controlar a dor leve/moderada em RN. Caso não seja possível sua disponibilidade, outros métodos como glicose a $25 \%$ ou sucção não nutritiva podem ser empregados. A eficácia de intervenções não farmacológicas na redução da dor já está comprovada em muitos estudos, ainda que não existam diretrizes definidas sobre qual método é superior [5].

Esses métodos não farmacológicos podem ser utilizados de forma individual como tratamento único diante de presença de dor leve, ou como intervenções de suporte (auxiliares) diante de situações de dor moderada a intensa. Evidências científicas comprovam a ação sinérgica e protetora dos métodos não farmacológicos quando utilizados de forma associada, 
sendo a sucção não nutritiva e a solução oral de glicose a combinação mais utilizada e eficaz. Quando houver recursos viáveis ??e adequados disponíveis, mediante procedimentos assistenciais dolorosos em neonatos, a equipe de saúde deve usar a combinação de métodos ambientais e não farmacológicos para obter analgesia ideal [3-5].

Conclui-se que tais intervenções apresentam gastos mínimos, são métodos de fácil aprendizado, com boa aceitação e execução pela equipe assistencial de saúde e praticamente não apresentam danos ou sequelas ao RN [5].

Elementos que influenciam a aplicação de medidas não-farmacológicas para alívio da dor de recém-nascidos em unidades de terapia intensiva neonatal

Existem certas dificuldades que limitam a utilização dessas intervenções em unidades neonatais: a falta de conhecimento dos pais e profissionais da saúde sobre as vantagens e efetividade destas técnicas; convicção por alguns profissionais desatualizados de que os recémnascidos poderão relacionar os estímulos dolorosos ao fenômeno da amamentação ou ao contato pele a pele; o déficit de conhecimento dos pais para a aplicação dessas medidas, como também a indispensabilidade de aprimorar a técnica da intervenção ao uso das medidas do alívio da dor são alguns dos fatores limitantes descritos na literatura científica [6].

Estudo apontou a dificuldade de acesso e falta de registros nos prontuários dos neonatos sobre as anotações das intervenções realizadas para alívio da dor. Adicionado a esse achado, temos pesquisas nacionais que foram desenvolvidas com profissionais da equipe de enfermagem que trabalhavam em unidades de terapia intensiva neonatal, onde grande parte da amostra alegou que não executam as anotações nos prontuários sobre as intervenções não farmacológicas que utilizaram, ou dificilmente relatam com dados percentis de aproximadamente $50 \%$, segundo as enfermeiras participantes do estudo, e de $20 \%$, segundo os auxiliares/técnicos de enfermagem. Os profissionais da saúde necessitam estar entusiasmados, buscando sempre se atualizar, respaldando seu trabalho através do registro adequado das suas ações no prontuário. Porém, esses registros apresentam-se limitados e muitas vezes ausentes devido à falta de conhecimento sobre essas intervenções, uma vez que os cuidados voltados para 0 manejo da dor não são valorizados e nem reconhecidos como um cuidado essencial $[7,8]$.

Os fatores dificultantes relatados pelos profissionais da saúde diante da prática do manuseio da dor que mais foram evidenciadas compreenderam os aspectos individuais, de trabalho coletivo, complexidade e dificuldade da assistência neonatal e institucional. A assistência direcionada ao manejo da dor é composta pela identificação dos sinais (físicos e comportamentais) de dor apresentados pelos neonatos, avaliações direcionadas através da utilização de escalas de dor e planejamento dos cuidados e tratamento adequado. Também foram relatados pelos profissionais de saúde a prioridade de realização de treinamento e capacitações sobre o manejo da dor neonatal devido à falta de conhecimento sobre a sua identificação, avaliação e tratamento, causando assim a incompatibilidade da sua prática [8].

Sobre os obstáculos direcionados ao sistema e organização institucional, foram relatadas a falta de rotinas assistenciais e protocolos voltados para avaliação e tratamento da dor do RN. Fatores ligados à carga horária dos profissionais de saúde como também o aumento de quantitativo de recursos humanos foram apontadas como limitações para a execução do manejo da dor. Diante destes dados, pode-se concluir que a carga horária excessiva dos profissionais de saúde que possuem mais de um vínculo de trabalho também é considerada como uma barreira para execução do tratamento da dor neonatal [8].

É necessário, portanto, que os profissionais de saúde, principalmente a equipe de enfermagem, tenham interesse em aderir ao uso de medidas e condutas para avaliação e controle da dor, como também realizar treinamentos de educação continuada sobre a dor neonatal, focando no processo de sensibilização profissional quanto à adesão e realização dos registros das ações práticas realizadas, além de intensificar o processo de comunicação e implementação da supervisão assistencial pela enfermagem [9].

Repercussões dos pais e neonatos diante da aplicação de medidas não-farmacológicas para alívio da dor

Dados de um estudo afirmam que, de acordo com a aplicação da escala de dor neonatal (NIPS - Neonatal Infant Pain Scale), após o procedimento de punção calcânea, os RN que receberam algum tipo de medidas não-farmacológicas apresentaram redução da face contraída, redução do tempo de choro vigoroso, redução dos escores de dor, movimento dos braços e 
pernas com os membros relaxados. No entanto, a regulação da frequência cardíaca e saturação de oxigênio não apresentaram diferenças significativas [10].

Sobre a inserção dos pais na aplicação das medidas para alívio da dor neonatal, um estudo relatou que eles tinham o desejo de aumentar o bem-estar de seus filhos. Eles relataram seu interesse em participar de forma ativa e presente durante os cuidados para aliviar a dor do bebê, apoiar, ajudar e pensar sobre o que era melhor para o neonato. Os pais sentiram que ver o aumento da reação positiva do RN, como a sensação de segurança e o seu bem-estar, aumentou a motivação dos pais para participar do alívio da dor dos seus filhos [11].

A motivação dos pais também foi associada ao crescimento do papel dos pais, como a formação do vínculo entre pais e filhos, experimentando a presença de alguém como importante e confiança nas próprias habilidades. Alguns pais achavam que sua própria atividade de cuidar do bebê e participar do alívio da dor era um fator contribuinte [11].

\section{Legislação sobre o manejo da dor neonatal}

A Declaração Universal de Direitos para o bebê prematuro aborda, no artigo IV, sobre os direitos que todos os prematuros têm ao tratamento estabelecido pela ciência, devendo receber cuidados por uma equipe multiprofissional capacitada, tendo como objetivo o bem-estar e desenvolvimento do neonato. $\mathrm{O}$ artigo $\mathrm{VI}$, por sua vez, alega que nenhum RN deve sofrer tortura ou receber tratamento cruel, desumano ou degradante; sempre a sua dor deve ser considerada, prevenida e tratada através dos métodos disponíveis pela ciência atual [12].

A American Academy of Pediatrics relata que qualquer indivíduo, independentemente da idade, tem o direito básico de ter o alívio da dor, embora a utilização de medidas efetivas para o manejo da dor neonatal ainda seja limitada. Diante disso, todos os profissionais da equipe de saúde que atuam na assistência direta ao recém-nascido devem ter como foco principal a prevenção e o tratamento da dor neonatal [2].

De acordo com o Manual do Método Canguru do Ministério da Saúde, o manejo da dor tem como objetivo a padronização dos métodos de controle e tratamento da dor neonatal. As principais intervenções para o alívio da dor são: transformar a UTIN em um ambiente acolhedor, respeitar o sono do RN, agrupar os cuidados com objetivo de evitar o manuseio excessivo, posicionamento adequado, estimular 0 apoio dos pais para realização das medidas nãofarmacológicas em conjunto com a equipe. As intervenções não-farmacológicas mais utilizadas são a sucção não nutritiva, soluções adocicadas, leite materno, associação da sucção não nutritiva e sacarose, posição canguru, contenção, enrolamento, holding e o toque terapêutico [13].

Para transpor essas barreiras, devem ser implementadas técnicas de capacitações e compartilhamento de conhecimento sobre a temática da dor, com o intuito de melhorar as práticas dos cuidados e manuseio da dor no ambiente de UTIN. Geralmente, as instituições governamentais e não governamentais criam e publicam protocolos e recomendações sobre os métodos sobre o manejo da dor. Diante de tais publicações, as instituições hospitalares ajustam esses protocolos e recomendações, levando em consideração a realidade do público alvo do seu setor e os recursos disponíveis [14].

O estudo aponta para a elaboração de novas pesquisas que analisem o comportamento de instituições hospitalares e o seu processo de trabalho voltados para o manuseio da dor, considerando-a como o quinto sinal vital. Além disso, deve-se realizar: criação de protocolos de dor neonatal e sua implementação nas unidades de cuidados, estratégias focadas na segurança do paciente, condutas éticas e bioéticas que respaldem sua assistência no manejo da dor neonatal, implementação no setor da educação e ensino sobre estratégias de identificação, avaliação e tratamento da dor nos currículos dos profissionais de saúde, objetivando novos conhecimentos e mudanças de atitudes, integrando teoria e prática [15].

Frente à necessidade de mudança na assistência neonatal, torna-se importante a reorganização das Unidades Neonatais, com vistas a proporcionar atendimento qualificado a este público, auxiliando na redução da dor, assim como, no incentivo de criação de protocolos para aplicação de medidas não-farmacológicas para alívio da dor. Reforçam-se as diversas fragilidades e limitações ao realizar o manejo adequado da dor neonatal.

Percebeu-se que dos materiais utilizados, nove foram artigos, uma declaração de especialistas e uma diretriz do Ministério da Saúde, sendo grande parte publicados em revistas ou periódicos da saúde. Destes, cinco possuem nível de evidência $2 \mathrm{C}$, quatro apresentam nível de evidência 5 e dois estudos possuem nível de evidência 1B, de acordo com a Oxford Centre for Evidence-Based Medicine [16]. 
A execução deste estudo possibilitou maior entendimento sobre as medidas nãofarmacológicas para alívio da dor dos neonatos em UTIN, através da análise dos aspectos contextuais que permeiam esse fenômeno, apresentando os métodos mais utilizados na prática neonatal, mostrando as perspectivas dos profissionais da saúde quanto às principais dificuldades encontradas para aplicação dessas intervenções, como também os aspectos vivenciados pelos pais e neonatos diante sua aplicação.

A partir da análise dos contextos, evidencia-se como principal limitação deste estudo o fato de ter sido analisado mediante o referencial teórico proposto, para tanto, é necessário realizar novas análises utilizando outros referenciais.

Para a enfermagem, pontua-se a importância de promover treinamentos e capacitações com a equipe sobre a identificação e manejo da dor neonatal, compreendendo os diversos aspectos que podem influenciar o alívio da dor. Também pontua a relevância do apoio dos pais junto ao cuidado multiprofissional durante a aplicação das medidas para alívio da dor.

Essas medidas visam a promoção de uma atenção integral à saúde centrada no indivíduo, visando uma reorganização dos serviços de saúde e implementação de protocolos para avaliação e tratamento da dor, tendo como base a integralidade do recém-nascido e considerando a dor como um sinal vital essencial a ser avaliado.

\section{Referências}

1. Bonutti DP, Daré MF, Castral TC, Leite AM, Maia JAV, Scochi CGS. Dimensioning of painful procedures and interventions for acute pain relief in premature infants. Rev Latinoam Enferm 2017;25:e2917. https://doi.org/10.1590/1518-8345.1387.2917

2. Keels E, Sethna N, Watterberg KL, Cummings JJ, Benitz WE, Eichenwald EC et al. Prevention and management of procedural pain in the neonate: an update. Pediatr Rev 2016;137(2):e20154271. https://doi.org/10.1542/peds.2015-4271

3. Stevens B, Yamada J, Ohlsson A, Haliburton S, Shorkey A. Sucrose for analgesia in newborn infants undergoing painful procedures. Cochrane Database Syst Rev 2016;7(7):CD001069. https://doi.org/10.1002/14651858.CD001069.pub5

4. Hinds $P$, Chaves $D$, Cypress $S$. Context as a source of meaning and understanding. Qual Health Res 1992;2(1):61-74. https://doi.org/10.1177/104973239200200105

5. Kumar P, Sharma R, Rathour S, Karol S, Karol MDCH. Effectiveness of various nonpharmacological analgesic methods in newborns. Clin Exp Pediatr 2020;63(1):2529. https://doi.org/10.3345/kjp.2017.05841

6. Harisson D, Bueno M, Reszel J. Prevention and management of pain and stress in the neonate. Res Rep Neonatol 2015;15(5):9-16. https://doi.org/10.2147/RRN.S52378

7. Soares ACO, Caminha MFC, Coutinho ACFP, Ventura CMU. Pain in the neonatal unit: the knowledge, attitude and practice of the nursing team. Cogitare Enferm 2016;21(2):01-10. https://doi.org/10.5380/ce.v21i2.42897

8. Christoffel MM, Querido DL, Silveira ALD, Magesti BN, Gomes ALM, Silva ACCS. Health professionals' barriers in the management, evaluation, and treatment of neonatal pain. BrJP. 2019;2(1):34-38. https://doi.org/10.5935/2595-0118.20190007

9. Sposito NPB, Rossato LM, Bueno M, Kimura AF, Costa T, Guedes DMB. Assessment and management of pain in newborns hospitalized in a Neonatal Intensive Care Unit: a cross-sectional study. Rev Latinoam Enferm 2017;25:e2931. https://doi.org/10.1590/1518-8345.1665.2931

10. Gomes PPS, Lopes APA, Santos MSN, Façanha SMA, Silva AVS, Chaves EMC. Nonpharmacological measures for pain relief in venipuncture in newborns: description of behavioral and physiological responses. BrJP 2019;2(2):142-6. https://doi.org/10.5935/2595-0118.20190026

11. Palomaa AK, Korhonen A, Pölkki T. Factors influencing parental participation in neonatal pain alleviation. J Pediatr Nurs 2016;31(5):519-27. https://doi.org/10.1016/i.pedn.2016.05.004

12. Tavares LAM. Uma declaração universal de direitos para o bebê prematuro. Rio de Janeiro: Diagraphic; 2009. Disponível em: http://www.aleitamento.med.br/upload\%5Carquivos\%5Carquivo1 2044.pdf 
13. Brasil. Ministério da Saúde. Secretaria de Atenção à Saúde. Departamento de Ações Programáticas Estratégicas. Método canguru: diretrizes do cuidado [Internet]. Brasília; 2018 [citado 2020 Set 20]. Disponível em:

http://bvsms.saude.gov.br/bvs/publicacoes/metodo canguru diretrizes cuidado revisa da.pdf

14. Maciel HIA, Costa MF, Costa ACL, Marcatto JO, Manzo BF, Bueno M. Pharmacological and nonpharmacological measures of pain management and treatment among neonates. Rev Bras Ter Intensiva 2019;31(1):21-6. https://doi.org/10.5935/0103$\underline{507 x .20190007}$

15. Christoffel MM, Castral TC, Daré MF, Montanholi LL, Gomes ALM, Scochi CGS. Attitudes of healthcare professionals regarding the assessment and treatment of neonatal pain. Esc Anna Nery Rev Enferm 2017;21(1):e20170018.

https://doi.org/10.5935/1414-8145.20170018 